\title{
Inhomogeneous Ferromagnetism and Unconventional Charge Dynamics in Disordered Double Exchange Magnets
}

\author{
Sanjeev Kumar and Pinaki Majumdar \\ Harish-Chandra Research Institute, \\ Chhatnag Road, Jhusi, Allahabad 211 019, India
}

(May 15, 2003)

\begin{abstract}
We solve the double exchange model in the presence of arbitrary substitutional disorder by using a self consistently generated effective Hamiltonian for the spin degrees of freedom. The magnetic properties are studied through classical Monte Carlo while the effective exchange, $D_{i j}$, are calculated by solving the disordered fermion problem, and renormalised self-consistently with increasing temperature. We present exact results on the conductivity, magnetoresistance, optical response and 'real space' structure of the inhomogeneous ferromagnetic state, and compare our results with charge dynamics in disordered $\mathrm{La}_{1-x} \mathrm{Sr}_{x} \mathrm{MnO}_{3}$. The large sizes, $\mathcal{O}\left(10^{3}\right)$, accessible within our method allows a complete, controlled calculation on the disordered strongly interacting problem.
\end{abstract}

The double exchange (DE) model provides the simplest example of strong coupling itinerant electron ferromagnetism, and is at the heart of the remarkable magnetotransport [1] in the manganites. The qualitative magnetic features of the model are reasonably well understood now [2], although transport properties have been much less explored. The problem is more difficult in the presence of quenched disorder, which arises inevitably in real materials [3-7] from ionic substitution and resulting bond distortions. Disorder leads to an inhomogeneous magnetic state and enhances the density of low energy spin fluctuations. The resistivity arising out of structural disorder and magnetic scattering is usually large, comparable to the Mott resistivity $[5,6]$, violates Mathiessens rule, and cannot be accessed by standard transport theory. The optical response reveals a strongly non Drude character [6,7], implying unconventional charge dynamics, and rapid loss in low energy spectral weight with rising temperature.

Most of these features, which depend explicitly on the inhomogeneous spatial character of the magnetic and electronic state cannot be captured within 'mean field' approximations, including 'dynamical mean field theory' (DMFT), and current 'real space' approaches are severely size limited in three dimension. In this paper we use a new [8] Monte Carlo (MC) technique, which handles the interplay of disorder and spin correlation essentially exactly, to provide the first controlled results on charge dynamics in the disordered double exchange (DDE) model. We map out the phase diagram, clarify the nature of the inhomogeneous ferromagnetic state, and provide results on magnetotransport and optical response in the model. Our results are directly relevant to the 'coherent to incoherent' crossover [5-7] in disordered $\mathrm{R}_{1-x} \mathrm{Sr}_{x} \mathrm{Mn}_{1-z} \mathrm{Al}_{z} \mathrm{O}_{3}$, and the properties of disordered metallic ferromagnets [9] in general. This approach can be readily extended to include phonon degrees of freedom, of key importance in manganite physics.
The DDE model has been proposed earlier to explain the ferro-metal (FM) to para-insulator (PI) transition, and the associated colossal magnetoresistance (CMR) in manganites. It is now accepted that electron-phonon coupling, and possibly phase coexistence, are important [1] in understanding CMR. While the DDE model can exhibit a FM-PI transition, at large disorder, in real materials even weak electron-phonon coupling will strongly affect the 'Anderson localised' PI phase. We will illustrate the FM-PI transition, but focus more on the 'metallic' phases, of relevance to the disordered $\mathrm{Re}_{1-x} \mathrm{Sr}_{x} \mathrm{MnO}_{3}$ family at $x \gtrsim 0.3$. In these materials phonon effects are not significant and the effect of spin fluctuations on diffusive charge dynamics can be directly probed.

We study the following model:

$$
H=\sum_{\langle i j\rangle} t_{i j} c_{i \sigma}^{\dagger} c_{j \sigma}+\sum_{i}\left(\epsilon_{i}-\mu\right) n_{i}-J_{H} \sum_{i} \mathbf{S}_{i} \cdot \vec{\sigma}_{i}
$$

The $t_{i j}=-t$ are nearest neighbour hopping on a cubic lattice and the on site disorder $\epsilon_{i}$ is distributed uniformly between $\pm \Delta / 2$. We set $J_{H} / t \rightarrow \infty$. The parameters in the problem are $\Delta / t$, and density $n$ (or chemical potential $\mu$ ). We set $t=1$, fixing our basic energy scale, and assume the core spins to be classical, with $\left|\mathbf{S}_{i}\right|=1$.

There are two key steps in solving for the magnetic and transport properties of a model like this: (i) Evaluate the correlated spin distribution, $P\left\{\mathbf{S}_{i}\right\}$, controlling the magnetic response, by 'integrating out' the electronic degrees of freedom. $\left\{\mathbf{S}_{i}\right\}$ denotes the full spin configuration. (ii) Solve for charge dynamics; resistivity, optical response, etc, in the background of structural disorder and equilibrium spin configurations.

Previous studies of the model have used variational mean field (VMF) theory [10] and DMFT $[11,12]$ to access the magnetism. Transport properties have been analysed within DMFT [11] and by using 'scaling theory' [13] in the limits of a spin polarised $(T=0)$ state and a fully spin disordered $\left(T \gg T_{c}\right)$ state. Finally, real 


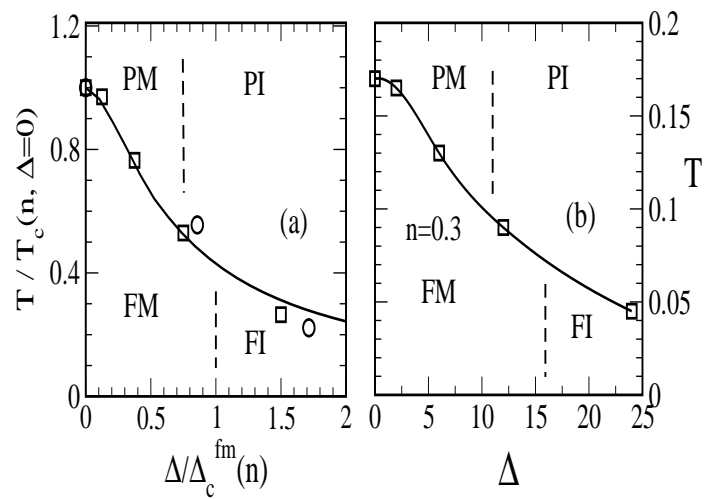

FIG. 1. (a). Approximate 'universal' phase diagram of the DDE model. For a specified electron density, $n, \Delta$ is measured in units of the $T=0$ critical disorder, $\Delta_{c}^{F M}(n)$, while $T$ is measured in units of $T_{c}(n, \Delta=0)$. Our data for $n=0.1$ (circles) and $n=0.3$ (squares) are described approximately by a common fit. (b). Actual phase diagram for $n=0.3$ The 'boundary' separating the PM and PI phases is a crossover.

space MC technique has been used [14] to study the magnetic transition and the spin wave spectrum. Both VMF and DMFT 'factor' the correlated spin distribution into a product of single site distributions. This loses out on spin correlations and trivialises the paramagnetic phase. In addition, DMFT misses out key vertex corrections in the 'disorder average' process, losing electron localisation effects. The 'scaling theory' approach to electronic transport is exact for $T \rightarrow 0$ and $T \gg T_{c}$, but not of much use in the crucial regime around $T_{c}$. The only approach which implements step $(i)$ exactly is Monte Carlo, but the computational cost of these simulations limits available sizes to $\sim 4^{3}$ in three dimension [15]. The small linear dimensions, and the large finite size gaps, make it impossible to reliably estimate transport properties. This is where our method, below, allows a breakthrough.

For $J_{H} / t \rightarrow \infty$, a standard transformation and projection [8] can be used to map on $H$ to a 'spinless fermion' problem with core spin dependent hopping amplitudes: $H \equiv-t \sum_{\langle i j\rangle} f_{i j}\left(e^{i \Phi_{i j}} \gamma_{i}^{\dagger} \gamma_{j}+h . c\right)+\sum_{i}\left(\epsilon_{i}-\mu\right) n_{i}$. The hopping amplitude $g_{i j}=f_{i j} e^{i \Phi_{i j}}$, between locally aligned states, can be written in terms of the polar angle $\left(\theta_{i}\right)$ and azimuthal angle $\left(\phi_{i}\right)$ of the spin $\mathbf{S}_{i}$ as, $\cos \frac{\theta_{i}}{2} \cos \frac{\theta_{j}}{2}$ $+\sin \frac{\theta_{i}}{2} \sin \frac{\theta_{j}}{2} e^{-i\left(\phi_{i}-\phi_{j}\right)}$. The 'magnitude' of the overlap, $f_{i j}=\sqrt{\left(1+\mathbf{S}_{i} \cdot \mathbf{S}_{j}\right) / 2}$, and the phase is specified by $\tan \Phi_{i j}=\operatorname{Im}\left(g_{i j}\right) / \operatorname{Re}\left(g_{i j}\right)$.

The fermions in this 'quadratic' problem move in the background of quenched disorder $\epsilon_{i}$ and 'annealed disorder' in the hopping amplitudes $g_{i j}$. To exploit the 'non interacting' character of the fermion problem we need to know the relevant $\{f, \Phi\}$ configurations, controlled by $H_{e f f}\{f, \Phi\}=-\frac{1}{\beta} \log T r e^{-\beta H}$. The corresponding Boltzmann distribution is $P\{f, \Phi\} \propto e^{-H_{e f f}\{f, \Phi\}}$. Our key proposal [8] is $-\frac{1}{\beta} \log T r e^{-\beta H} \approx-\sum_{\langle i j\rangle} D_{i j} f_{i j}$ The effective 'exchange' $D_{i j}$, in the short range classical spin model, is determined self consistently as the thermal average of the 'mixed' spin-fermion operator, $\hat{\Gamma}_{i j}=$ $\left(e^{i \Phi_{i j}} \gamma_{i}^{\dagger} \gamma_{j}+h . c\right)$ over the assumed equilibrium distribution. The self-consistent $D_{i j}$ are solved for via $\mathrm{MC}$ for a specified $\mu,\left\{\epsilon_{i}\right\}$, and $T$. The $D_{i j}$ can be spatially strongly inhomogeneous and also significantly temperature dependent. At self-consistency fermionic averages are computed over equilibrium spin configurations. We work at constant $n$, fixing $\mu$ through iteration. Since we avoid the expensive spin update procedure of 'exact' $\mathrm{MC}$ and use diagonalisation only to compute the $D_{i j}$ we can access sizes $\sim 10^{3}$, compared to $4^{3}$ in the standard approach. Transport properties, at equilibrium, are computed exactly using the Kubo formula, employing sizes $\sim 8^{3}-10^{3}$. We systematically check for size dependence in our transport results. The conductivity results are in units of $\left(\pi e^{2}\right) / \hbar a_{0}$, the Mott limit being $\left(0.03 e^{2}\right) / \hbar a_{0}$.

Fig. 1 shows the "global" phase diagram of the DDE model. We have studied the problem at $n=0.3$ and $n=0.1$, varying disorder from the perturbative end to the localisation regime. Fig.1.(a). superposes the results at $n=0.3$ and $n=0.1$, appropriately scaling the disorder and temperature (see caption). The critical disorder for the PM-PI crossover, or the disorder dependence of $T_{c}$ need not be 'universal' but seems to follow the same overall trend at moderate $n$. Fig.1.(b) shows the 'true' phase diagram specifically at $n=0.3$. The $T_{c}$ of $\mathrm{DE}$ models is approximately related to the internal energy change: $T_{c} \log (2 S+1) \sim \mathcal{E}\left(T_{c}\right)-\mathcal{E}(0)$, which in turn is related to the kinetic energy, $K$, at $T=0$. At small $\Delta$ and $T=0, K(\Delta) \sim K(0)-\chi_{d} \Delta^{2}$, where $\chi_{d}$ is the 'local' density response function. At strong disorder, in the 'localised' phase, $K(\Delta) \propto t^{2} / \Delta$. The correspondence of these limits with the inferred $T_{c}(\Delta)$ is visible in Fig.1.(b).
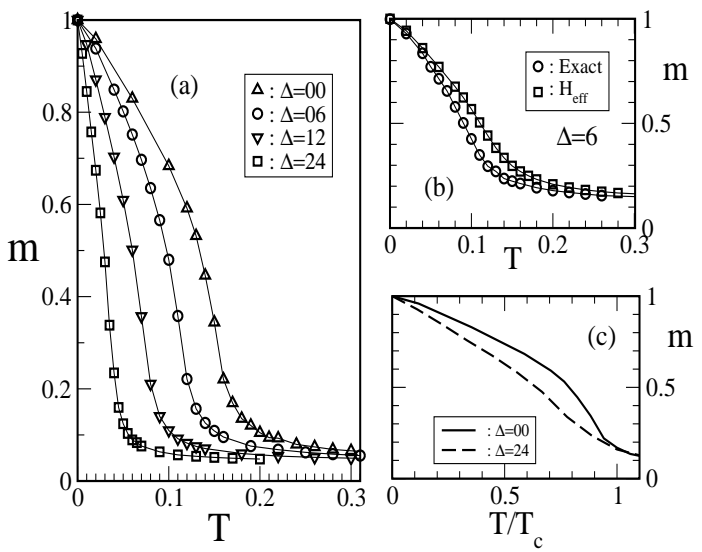

FIG. 2. (a). Temperature dependence of magnetisation at $n=0.3$, with $\Delta$ increasing from $0-24$. The inferred $T_{c}(\Delta)$ is shown in Fig.1. (b). Comparison of $m(T)$ obtained via exact $\mathrm{MC}$ and $H_{\text {eff }}$ on a $4 \times 4 \times 4$ system at $n=0.3$. Disorder average over 8 realisations in both case. $(c)$. The scaled magnetisation $m\left(T / T_{c}(\Delta)\right)$, at $n=0.3$, for $\Delta=0$ and $\Delta=24$. 


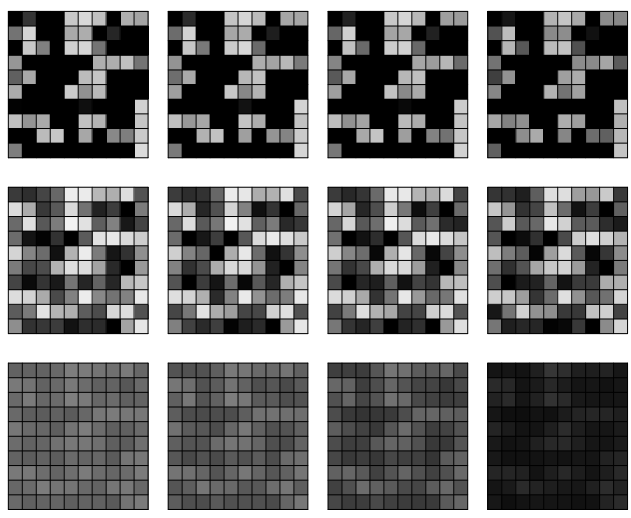

FIG. 3. Real space images, top surface of a $10 \times 10 \times 10$ cube with $\mathrm{PBC}$, at $n=0.3$ and $\Delta=6$, for a specific realisation of disorder. First row: electron density distribution, $\langle n(\mathbf{r})\rangle_{T}$, second row: effective exchange, $D(\mathbf{r}, \mathbf{r}+\delta: T)$, third row: nearest neighbour spin correlation, $f_{2}=\langle\mathbf{S}(\mathbf{r}) \cdot \mathbf{S}(\mathbf{r}+\delta)\rangle_{T}$. Temperatures along the row are, $T / T_{c} \sim 1.2,0.9,0.6$ and 0.2. Dark regions correspond to high density (top row), strong exchange (central row), and strong FM correlation (bottom).

Note that even at strong localisation, $\Delta \sim 24$, local excursion of the electrons can still sustain a $T_{c} \sim 0.05$, which would be $\sim 75 \mathrm{~K}$ for $t \sim 150 \mathrm{meV}$. DMFT and VMF also provide qualitatively similar trends in $T_{c}$.

Fig.2.(a) shows $m(T)$ with increasing disorder, at $n=$ 0.3, from which the data in Fig.1.(b) was inferred, while Fig.2.(b) compares the $m(T)$ computed with $H_{\text {eff }}$ with the 'exact' $\mathrm{MC}$ at $\Delta=6$. Although the asymptotic behaviour of $T_{c}(\Delta)$ is easy to motivate, Fig.2.(c) provides the first indication that the properties of the disordered ferromagnet cannot be understood by merely scaling $T_{c}$. Even though the $T=0$ state is fully polarised, at low finite $T$ the 'weak' bonds lead quickly to local disordering. The magnetisation $m\left(T / T_{c}\right)$ falls more sharply at low temperature in the disordered system [9]. The inhomogeneous character, and correspondingly wide distribution, of the 'exchange' leads to an overall increase in the density of low energy magnetic excitations. In addition to suppressing $m(T)$ it enhances the specific heat, $C_{V}\left(T / T_{c}\right)$, and reduces the spin wave stiffness $D_{S W}$.

The real space images, Fig.3, illustrate the inhomogeneous freezing into a ferromagnetic state in a 'cooling sequence', left to right. The density profile $\langle n(\mathbf{r})\rangle$, first row, remains unchanged over the $T$ range of interest, $0-1.2 T_{c}$. The exchange, $D_{i j}(T)$, central row, is strongly spatially inhomogeneous, but as a whole not strongly $T$ dependent. The bond distribution, $P(D, T)$, reveals [16] that weak bonds at low $T$ get quickly weakened with increasing $T$ while strong bonds are essentially $T$ independent. The nearest neighbour spin correlation, $f_{2}$, highlights the inhomogeneous disordering of the polarised state with increasing $T$ (white regions, two panels on the right), and the surviving local order for $T>T_{c}$ (left panel).

These results suggest the possible correlation between spatial inhomogeneity in the magnetic state and the bulk thermodynamics. The interplay of thermal spin disorder and 'frozen' structural disorder also affects the charge dynamics. Increasing $\Delta$ increases the residual resistivity, with $\rho_{0} \sim \rho_{\text {Mott }}$ at $\Delta \sim 10$. The resistivity in the paramagnetic phase $\left(\rho_{\text {inf }}\right)$, tracked at $T=0.4$ in Fig.4.(b), is not simply the additive contribution of structural and spin disorder. If Mathiessens rule were obeyed, $\rho_{\text {inf }}-\rho_{0}$ should have been constant. The deviation arises from interference between structural and magnetic scattering and is clearly observed in the metallic $\mathrm{Re}_{1-x} \mathrm{Sr}_{x} \mathrm{MnO}_{3}$, at $x=0.4$, with Re being, La, $\mathrm{Pr}$, Nd [6], and in $\mathrm{La}_{1-x} \mathrm{Sr}_{x} \mathrm{Mn}_{1-z} \mathrm{Al}_{z} \mathrm{O}_{3}$, at $x=0.3$, varying $z$ [5]. This 'interference' is beyond Boltzmann theory. With $a_{0} \sim 4 \AA, \rho_{M o t t} \sim 5 \mathrm{~m} \Omega \mathrm{cm}$ in the manganites. For the 'disordered' LaSr family, as $\rho_{0} / \rho_{\text {Mott }}$ varies from $0.005-0.04$ with increasing disorder, the corresponding $\rho_{\text {inf }} / \rho_{\text {Mott }}$ increases from $0.4-1.5[5,6]$.

Fig.4.(a) shows the normalised resistivity $\delta \rho(T)=$ $(\rho(T)-\rho(0)) /(\rho(0.4)-\rho(0))$ for $\Delta=0,2,6$. The shift in $T_{c}$ in this disorder regime is quite small, but $\delta \rho\left(T / T_{c}\right)$ quickly changes character with increasing disorder. The more prominent short range spin fluctuations in the disordered system couple to the diffusive electrons leading to a sharper rise in $\rho(T)$. While most of the rise in the 'clean' system occurs in the vicinity of $T_{c}$, the rise is spread over a wide interval in the disordered system.

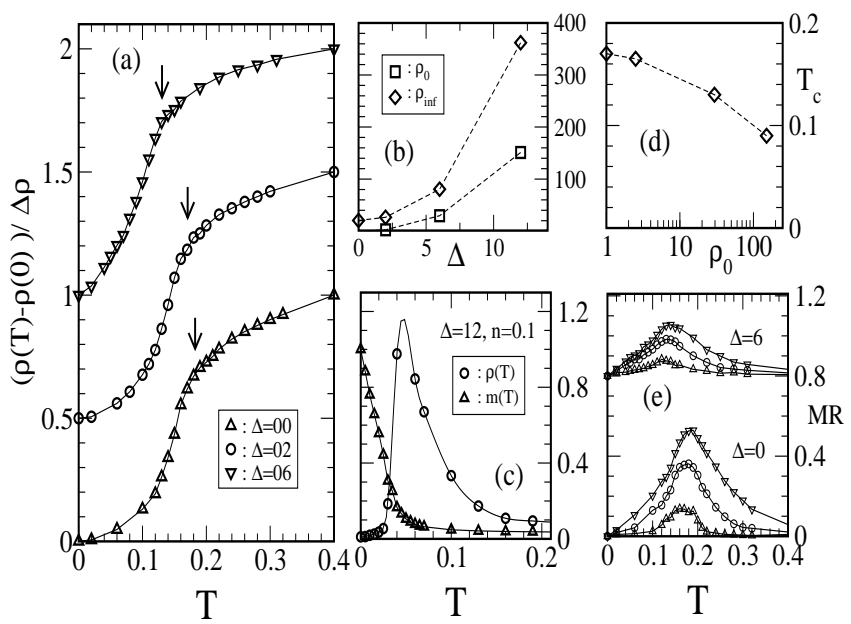

FIG. 4. (a). Temperature dependence of resistivity at $n=0.3$. We plot $\rho(T, \Delta)-\rho(0, \Delta)$ normalised by $\rho(0.4, \Delta)-\rho(0, \Delta)$, shifting successive curves by 0.5 for clarity. $T_{c}$ marked by an arrow on each curve. (b). Resistivity at $T=0, \rho_{0}$, and at $T=0.4, \rho_{\text {inf }}$, with increasing disorder. Note the clear violation of Mathiessens rule even at $\Delta=2$ (see text). (c) Resistivity normalised to maximum value $\left(\sim 10^{4}\right)$, and $m(T)$ at $n=0.1$ and $\Delta=12$ illustrating the FM $\rightarrow$ PI transition. $(d)$. Variation in $T_{c}$ with residual resistivity. Note the logarithmic $x$ scale. $(e)$. The magnetoresistance $(\rho(T, 0)-\rho(T, h)) / \rho(T, 0)$ at $n=0.3: \Delta=0$ (lower set) and $\Delta=6$ (upper set). The $\Delta=6$ set has been vertically shifted by 0.8 . Field values are $h=0,0.02,0.05,0.10$. 


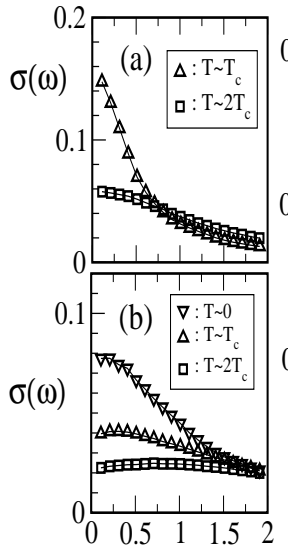

$\omega$

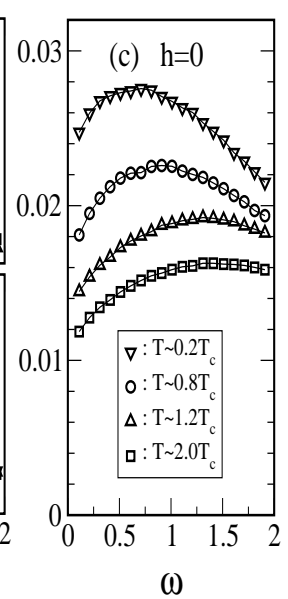

$\omega$

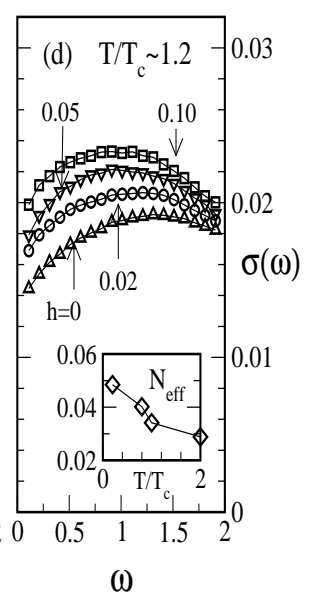

$\omega$
FIG. 5. Optical conductivity at $n=0.3 . \quad(a) . \quad \Delta=0$, (b). $\Delta=4,(c) . \quad \Delta=6$, at $h=0$, and $(d) . \Delta=6$ at $T \sim T_{c}$. Other parameters indicated in the figure. Inset in panel $(d)$ shows the integrated spectral weight, $N_{\text {eff }}(T)$, (see text) derived from the data in panel $(c)$. Data averaged over $4-8$ realisations of disorder.

Fig.4.(c). shows data at $n=0.1$ and $\Delta=12$ to illustrate the FM-PI transition. We work with this lower density because the MIT is easier to access. We solve the full problem for sizes $6 \times 6 \times L$, with $L=\{6,12,24\}$, disorder average, and extrapolate the computed 'd.c conductivity' to $L \rightarrow \infty$. This is crucial to capture 'Anderson localisation' in finite systems. The transport in the insulating phase is controlled by activation to the mobility edge.

The correlation between $T_{c}$ and residual resistivity has been experimentally explored [3,4] and Fig.4.(e) highlights the fall in $T_{c}$ as $\rho_{0}$ heads towards the Mott limit.

Our results on MR, Fig.4.(e), indicate that in the intermediate disorder regime the MR is actually smaller in the disordered problem than in the clean system. The MR is $1-\rho(T, h) / \rho(T, 0)$. The minimum $\rho(T, h)$ is limited by $\rho_{0}$, the residual resistivity. With increasing $\Delta, \rho_{0}$ grows, limiting the MR. The MR rises again only when $\Delta$ is large enough to drive a finite $T$, FM-PI transition.

Finally, the optical response of the system, at $n=0.3$ and $\Delta=0,4$ and 6 , is shown in Fig.5. Panel $(a)$. shows $\sigma(\omega)$ at $\Delta=0$ and the Drude feature survives even for $T \sim 2 T_{c}$ with $\sigma_{d c} \sim 6 \sigma_{M o t t}$. For $\Delta=4$, however, panel (b). reveals that $\sigma(\omega)$ changes from a Drude form for $T \lesssim T_{c}$ to an essentially 'flat' incoherent response at $T \sim 2 T_{c}$. At $2 T_{c}, \sigma_{d c} \sim 2 \sigma_{M o t t}$. This response is roughly like $\mathrm{La}_{1-x} \mathrm{Sr}_{x} \mathrm{MnO}_{3}$ at $x \sim 0.4$ [6]. At even larger disorder, $\Delta=6$, the response is non Drude even at $T=0$, and becomes markedly so, with a finite $\omega$ peak, as $T$ is increased. The apparently $\sqrt{\omega}$ rise results from the intimate coupling of the diffusive electrons to spin fluctuations via the hopping modulation. For $\Delta=6, \sigma_{d c} \sim$ $2 \sigma_{M o t t}$ at $T=0$, and $\sigma_{d c} \sim \sigma_{M o t t}$ at $T \sim 2 T_{c}$. This is like the response in $(\mathrm{Pr}, \mathrm{Nd})_{1-x} \mathrm{Sr}_{x} \mathrm{MnO}_{3}$ at $x \sim 0.4$ [6]. We predict that the response will be similar at $x=0.4$ in $\mathrm{La}_{1-x} \mathrm{Sr}_{x} \mathrm{Mn}_{1-z} \mathrm{Al}_{z} \mathrm{O}_{3}$ at $z \sim 1 \%-2 \%$. The inset to panel $(d)$. shows the integrated low frequency spectral weight $N_{\text {eff }}\left(\omega^{\prime}, T\right)=\int_{0}^{\omega^{\prime}} \sigma(\omega, T) d \omega$, at $\omega^{\prime}=2$. There is a $40 \%$ loss in spectral weight in the $T$ range $0-2 T_{c}$. Our energy cutoff in $N_{\text {eff }}$ roughly corresponds to $\omega^{\prime} \sim 0.3 \mathrm{eV}$ in the manganites. Panel $(d)$. shows the magneto-optical response at $\Delta=6$ and $T \sim 1.2 T_{c}$.

The non Drude relaxation with $\sigma(\omega)$ having a finite $\omega$ peak occurs in a regime where $d \rho / d T>0$, as in conventional metals, but the charge dynamics is highly diffusive as expected in a system with strong 'effective disorder'. The change from 'coherent' to 'incoherent' dynamics occurs in $\sigma(\omega, T)$ when $\rho_{d c}(T) \sim \rho_{\text {Mott }}$. This general feature is true of the PM phase of all the manganites.

In conclusion, we have discussed the inhomogeneous magnetism in disordered double exchange magnets, and provided the first "exact" results on transport and optical response. The dependence of the non Drude relaxation on disorder and temperature is fully consistent with the metallic manganites. The extension of our method to include phonons will allow a complete solution of charge response in the manganites.

We acknowledge use of the Beowulf cluster at H.R.I.

[1] See, e.g, E. Dagotto et al., : Phys Rep 344, 1 (2001); Colossal Magnetoresistive Oxides, Ed. Y. Tokura, Gordon \& Breach (2000).

[2] M. Yamanaka et al., Phys. Rev. Lett. 81, 5604 (1998); E. Dagotto et al., Phys. Rev. B 58, 6414 (1998); M. J. Calderon and L. Brey, Phys. Rev. B 58, 3286 (1998); K. Nagai et al., J. Phys. Soc. Jpn. 69, 1837 (2000).

[3] J. M. D. Coey et al., Phys. Rev. Lett. 75, 3910 (1995).

[4] L. M. Rodriguez-Martinez and J. P. Attfield, Phys. Rev. B 54, 15622 (1996).

[5] Y. Sawaki et al., Phys. Rev. B 61, 11588 (2000).

[6] E. Saitoh, et al., Phys. Rev. B 60, 10362 (1999).

[7] K. Takenaka et al., Phys. Rev. B 65,

[8] S. Kumar and P. Majumdar, cond-mat 0305345.

[9] T. Kaneyoshi, Introduction to Amorphous Ferromagnets, World Scientific, Singapore (1992).

[10] E. E. Narimanov and C. M. Varma, Phys. Rev. B 65, 024429-1 (2001).

[11] B. M. Letfulov and J. K. Freericks, Phys. Rev. B 64, 174409 (2001).

[12] M. Auslender and E. Kogan, Phys. Rev. B 65, 012408 (2001).

[13] L. Sheng, et al., Phys. Rev. Lett. 79, 1710 (1997).

[14] Y. Motome and N. Furukawa, J. Phys. Chem. Solids, 63, 1357 (2002).

[15] Some algorithms allow larger sizes: J. L. Alonso et al., Nucl. Phys. B 596, 587 (2001); Y. Motome and N. Furukawa, cond-mat 0305029.

[16] S. Kumar and P. Majumdar, to be published. 